\title{
Study on Transient Signals Recognition in Power System Based on Multiwavelet Packet Coefficient Entropy and Artificial Neural Network
}

\author{
Dongmin Li Zhigang Liu Baichao Huo Yuxiang Su \\ Institute of Electrification \& Automation, Southwest Jiaotong University, Chengdu 610031, P. R. China
}

\begin{abstract}
Multiwavelets own better properties than those of traditional wavelets. In the paper multiwavelet packet coefficient entropy (MPCE) is defined through combining decomposition coefficient of multiwavelet packet with entropy. A novel transient signals recognition method based on MPCE and artificial neural network (ANN) is proposed. Firstly, the appropriate multiwavelet packet decomposition of the sampled transient current signal is performed and each MPCE of transient current is calculated. Then eigenvector of multiwavelet packet of the current signal is constructed, and by taking the eigenvector as training samples the radial basis function (RBF) neural network is trained to implement the transient signals recognition. At last the proposed method is compared with the means based on traditional wavelet packet and ANN. Simulation results show that the proposed method is effective and feasible and the recognition capability is better than the method based on traditional wavelet packet and ANN.
\end{abstract}

Keywords: Multiwavelet packet, Traditional wavelet packet, RBF neural network, Multiwavelet packet coefficient entropy

\section{Introduction}

Detecting and recognition transient signals are widely used in power system, such as fault detecting and classification, relays protection, power quality assessment, fault location, equipment condition monitoring, and transient stability analysis. Effective classification of transient signal leads to quick and reliable transient protection operation.

Many theories, like wavelet analysis, mathematical morphological, support vector machine, have been introduced into power system transient signal detecting and identifying. Much fruitful work have been gained in this field, especially in fault phase selection, power quality disturbance, lighting strokes and temporary and permanent fault [1]-[2]. However, to the whole power system, there are still many issues in recognition of certain signals and environments.

Multiwavelets can own symmetry, orthogonality, short support and high order vanish moments; however, traditional wavelet cannot possess all these properties at the same time. Multiwavelets have been applied in power system [3]-[6]. In addition, there is more information on low and high frequency with multiwavelet decomposition than traditional wavelets. Multiwavelet packet is the extension of multiwavelets. And it has more high frequency information than multiwavelets do. In [7] de-noising and compression of fault signals in power system with multiwavelet packet has been studied. Multiwavelet packet has been tried in image compression in [8]. In [9] multiwavelet packet is used to diagnose the fault of gearbox. Multiwavelet packet possessing better properties than traditional wavelet packet can withdraw more abundant fault characteristics.

A transient signals recognition method can be treated as a problem of input-data pattern recognition and pattern-recognition problems can be well handled by artificial neural networks (ANNs). Recently, ANN has gained success in many power applications [10]-[12]. Many advantages are inherent in ANNs, including the excellent noise immunity and robustness, making their use less susceptible to operating conditions than conventional approaches. In this paper, RBF neural networks are used to identify each transient signal.

According to the feature of different transient signals in power system, and thinking about utilizing fully the abundant fault characteristics multiwavelet packet provides, a novel transient signals recognition method based on multiwavelet packet coefficient entropy and artificial neural network is proposed. At the same time the proposed method is compared with the means based on traditional wavelet packet and ANN. 


\section{The definition of multiwavelet packet coefficient entropy}

\subsection{Multiwavelet packet}

Wavelets have an associated scaling function $\phi(t)$ and wavelet function $\psi(t)$, but multiwavelets have two or more scaling and wavelet functions. The set of scaling functions can be written using the vector notation $\Phi(t)=\left[\phi_{1}(t) \phi_{2}(t) \cdots \phi_{r}(t)\right]^{T}, \quad$ where $\Phi(t)$ is called multi-scaling function. Likewise, the multiwavelet function is defined from set of wavelet function as $\Psi(t)=\left[\psi_{1}(t) \psi_{2}(t) \cdots \psi_{r}(t)\right]^{T}$, where $r$ means the multiplicity of multiwavelets, and $r \geq 2$. Multi-scaling function $\Phi(t)$ satisfied following two-scale matrix equation:

$$
\Phi(t)=\sum_{k=0}^{M} H_{k} \Phi(2 t-k)
$$

Multiwavelet function $\Psi(t)$ satisfies following two-scale matrix equation:

$$
\Psi(t)=\sum_{k=0}^{M} G_{k} \Phi(2 t-k)
$$

Where $H_{k}$ and $G_{k}(k \in[0, M], M \in Z)$ are $r \times r$ impulse response constant matrix.

The construction of multiwavelet packet is similar to wavelet packet. The multiwavelet filter bank procedure involves iterating the filtering operation on the low pass channel of the filter bank. Just as with scalar wavelets, new basis functions can be produced by iterating on the high pass channels of multiwavelet filter banks too. This new approach combines wavelet packet decomposition with multiwavelet decomposition.

Multiwavelet packet in a manner analogous to the definition of wavelet packet is defined.

Let $U_{0}(t)=\Phi(t)$ and $U_{1}(t)=\Psi(t)$.

Define

$$
\begin{aligned}
& U_{2 n}=\sum_{k=-\infty}^{\infty} H_{k} U_{n}(2 t-k) \\
& U_{2 n+1}=\sum_{k=-\infty}^{\infty} G_{k} U_{n}(2 t-k)
\end{aligned}
$$

From the space decomposition of multiwavelet packet, the reconstruction formula is below:

$$
U_{j, n}=\sum_{k}\left(H_{k}^{*} U_{j-1,2 n}+G_{k}^{*} U_{j-1,2 n+1}\right)
$$

Where $H_{k}^{*}$ and $G_{k}^{*}$ are respective conjugated transpose matrix of $H_{k}$ and $G_{k}$.

\subsection{Multiwavelet packet decomposition}

The frame of multiwavelet packet three layers decomposition is shown in Fig. 1, where we suppose the multiplicity of multiwavelets to be 2 . For multiwavelet, the choice of pre-processing method is one of most important problems. The problem of pre-processing methods still exists in multiwavelet packet. Multiwavelet packet decomposition involves the pre-processing of original sampled signals. Different pre-processing methods result in the application effect of multiwavelet packet. In [5] the optimum pre-processing method of each widely-used multiwavelet basis is studied in depth.

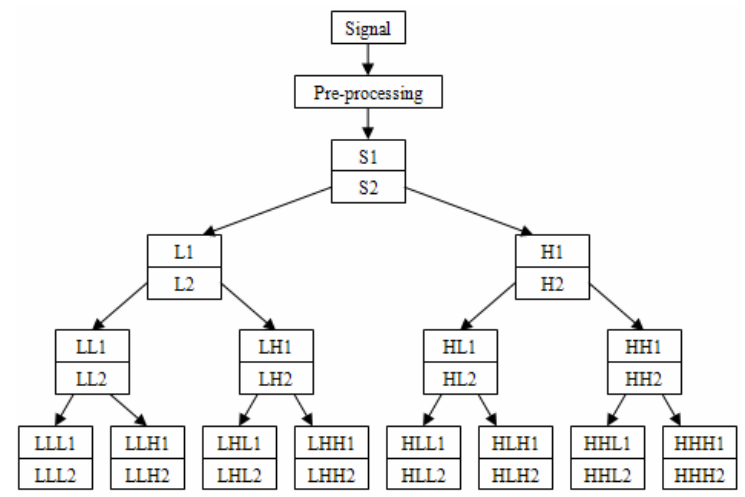

Fig. 1: Frame of multiwavelet packet decomposition.

As shown in the Fig. 1, the original sampled signal is decomposed into signals of eight frequency bands, and the signal formation of each frequency band is matrix. Compared with traditional wavelet packet decomposition, multiwavelet packet can withdraw more abundant and refined fault features from original fault signals.

\subsection{The definition of multiwavelet packet coefficient entropy}

As same as physical entropy, information entropy is used to describe uncertainty and complexity degree of systems. In recent years, Entropy has gained some achievements in power system [13]-[14]. Information entropy defined by Shannon may be described as the following [14]:

The uncertainty of any event is associated with its states and probabilities. The set of all possible states is called sample space $X,\left\{x_{1}, x_{2}, \cdots, x_{n}\right\}$. Each piece of information $x_{i}$ has a probability $P\left(x_{i}\right)=p_{i}$, 
$0 \leq p_{i} \leq 1, \sum p_{i}=1$. The entropy of $X$ is denoted by $H(X)$.

$$
H(X)=-\sum_{i=1}^{L} p_{i} \ln p_{i}
$$

When the uncertainty of each event is equal, the entropy has max value. The entropy of any certain event is zero. Therefore, entropy is the measurement of the uncertainty.

Through $i$ layers multiwavelet packet decomposition, a sequence of coefficient matrices $s_{i, j}\left(j=0 \sim 2^{i}-1\right)$ are obtained. These matrices are a kind of dividing forms of original signals. Then these coefficient matrices are transformed into probability distribution sequences. These sequences can reflect the sparse degree of the coefficient matrices. So the transformation formula of coefficient matrices is defined as the following:

$$
\varepsilon_{i, j, m}(k)=\frac{\left|S_{i, j, m}(k)\right|^{2}}{\sum_{k=1}^{N}\left|S_{i, j, m}(k)\right|^{2}}
$$

Where

$S_{i, j, m}(k)$ is the $k$ th value of sequence $S_{i, j, m}$;

$N$ is the length of sequence $S_{i, j, m}$;

$m$ is the dimension of coefficient matrices, in this paper $\mathrm{m}=1,2$.

Then according to fundamental principle of information entropy, through combining multiwavelet packet decomposition coefficient probability distribution sequences with information entropy multiwavelet packet coefficient entropy (MPCE) is defined.

$$
H_{i, j, m}=-\sum_{k=1}^{N} \varepsilon_{i, j, m}(k) \log \varepsilon_{i, j, m}(k)
$$

Where $H_{i, j, m}$ is the $m$ th dimension MPCE of the $j$ th frequency band of the ith layer decomposition.

\section{Feature extraction based MPCE}

\subsection{The model of transmission lines}

The EMTDC simulation model of a $500 \mathrm{kV}$ transmission line is set up as Fig. 2. Bergeron model is adopted for transmission lines. Parameters are: Positive sequence parameters $r_{1}=0.035 \Omega / \mathrm{km}, x_{1}=0.424 \Omega / \mathrm{km}$, $\mathrm{b}_{1}=2.726 \times 10^{-6} \mathrm{~S} / \mathrm{km}$; Zero sequence parameters $\mathrm{r}_{0}=0.3 \Omega / \mathrm{km}, \mathrm{x}_{0}=1.143 \Omega / \mathrm{km}, \mathrm{b}_{0}=1.936 \times 10^{-6} \mathrm{~S} / \mathrm{km}$. On this transmission line model there are 11 transient signals: 1-phase-to-ground (AG, BG and $\mathrm{CG}$ ), 2-phase-to-ground (ABG, BCG and $\mathrm{CAG}$ ), phase-to-phase $(\mathrm{AB}, \mathrm{BC}$ and $\mathrm{CA})$ and 3-phase $(\mathrm{ABC})$ short currents and lightning current. Sampling rate is $100 \mathrm{kHz}$.

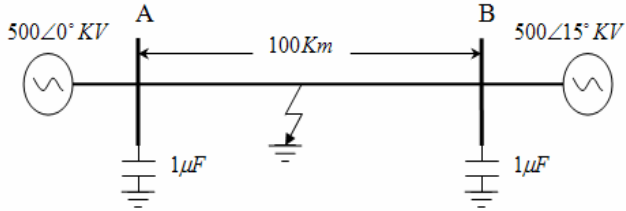

Fig. 2: Model of $500 \mathrm{kV}$ transmission line.

\subsection{The model of lightning current}

The lightning current waveform, which is influenced by some stochastic factor, has uncertain amplitude, wave front and wave tail, etc. However, investigations show that the Heidler function can represent the lighting current waveform [15].

$$
i(t)=\frac{I_{0}}{\eta} \frac{\left(t / T_{1}\right)^{2}}{1+\left(t / T_{1}\right)^{2}} e^{-t / T_{2}}
$$

Where $I_{0}$ is the maximum value of the current, $\eta$ is correction factor of the current peak, $T_{1}$ and $T_{2}$ are time constants determining current rise-time and current decay-time, respectively. In Fig. 3 the time to front $t_{f}$ and half peak $t_{h}$ of a lightning current is depicted. In addition, IEC regards $1.2 / 50 \mu s\left(t_{f}=1.2 \mu s, t_{h}=50 \mu s\right)$ as the standard lightning waveform parameters in lightning impulse experimentation.

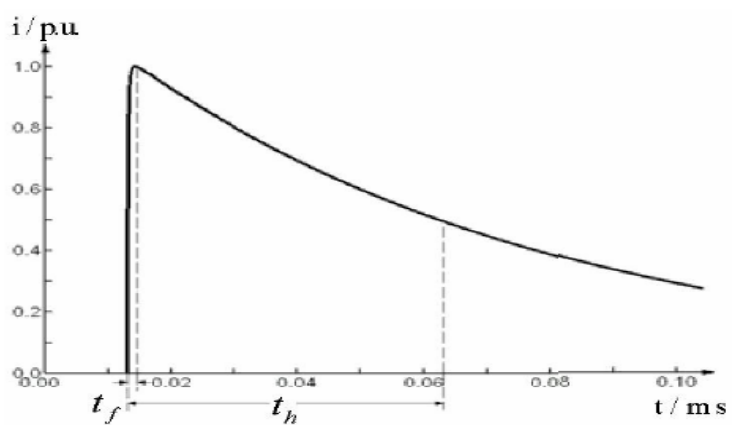

Fig. 3: Lightning current waveform of Heidler function $1.2 / 50 \mu \mathrm{S}$ 


\subsection{MPCE feature extraction of transient signals}

GHM multiwavelet packet with GHM.init [5] pre-processing method is adopted to implement three layers decomposition of sampled fault currents resulted from the EMTDC simulation. Data window width is half power cycle of pre-fault and half power cycle of post-fault. The MPCEs of each phase current of two fault types and normal operation are calculated according to (7) (8). They are shown by Table 1 where the faults take place at $65 \%$ distance of line away from bus-bar A at 0.2 second in Fig. 2.

\begin{tabular}{|c|c|c|c|c|c|c|c|c|c|}
\hline \multicolumn{2}{|c|}{ Fault types } & $\mathrm{H}_{3,0}$ & $\mathrm{H}_{3,1}$ & $\mathrm{H}_{3,2}$ & $\mathrm{H}_{3,3}$ & $\mathrm{H}_{3,4}$ & $\mathrm{H}_{3,5}$ & $\mathrm{H}_{3,6}$ & $\mathrm{H}_{3,7}$ \\
\hline \multirow{6}{*}{$\mathrm{AG}$} & \multirow{2}{*}{ Phase A } & 2.6880 & 2.4270 & 1.4270 & 0.1633 & 2.4641 & 0.1033 & 0.9247 & 1.0497 \\
\hline & & 2.6896 & 0.3954 & 0.0861 & 0.1525 & 0.1089 & 0.1434 & 0.1587 & 0.0933 \\
\hline & \multirow{2}{*}{ Phase B } & 3.1492 & 1.3313 & 0.5431 & 0.4193 & 1.0178 & 0.3616 & 0.5927 & 0.7357 \\
\hline & & 3.1367 & 0.0339 & 0.0406 & 0.2740 & 0.0603 & 0.5550 & 0.0307 & 0.1778 \\
\hline & \multirow{2}{*}{ Phase C } & 3.1384 & 1.0377 & 0.5203 & 0.3639 & 0.8465 & 0.3258 & 0.5359 & 0.7109 \\
\hline & & 3.1177 & 0.0328 & 0.0328 & 0.4131 & 0.0501 & 0.5109 & 0.0220 & 0.1652 \\
\hline \multirow{6}{*}{$\mathrm{AB}$} & \multirow{2}{*}{ Phase A } & 2.6889 & 0.5521 & 0.5724 & 0.6811 & 0.4993 & 0.5570 & 0.0928 & 0.1202 \\
\hline & & 2.7011 & 0.6680 & 0.4942 & 0.6262 & 0.6354 & 0.1395 & 0.3175 & 0.5999 \\
\hline & \multirow{2}{*}{ Phase B } & 2.6784 & 0.6894 & 0.6726 & 0.7421 & 0.5589 & 0.6433 & 0.1319 & 0.1679 \\
\hline & & 2.6962 & 0.5877 & 0.3750 & 0.7158 & 0.6892 & 0.1949 & 0.2310 & 0.6893 \\
\hline & \multirow{2}{*}{ Phase C } & 3.1587 & 0.8941 & 0.0805 & 0.0154 & 0.3269 & 0.0020 & 0.0219 & 0.0801 \\
\hline & & 3.1405 & 0.0036 & 0.0069 & 0.0283 & 0.0047 & 0.0191 & 0.0111 & 0.0427 \\
\hline \multirow{6}{*}{$\begin{array}{c}\text { Normal } \\
\text { operation }\end{array}$} & \multirow{2}{*}{ Phase A } & 3.1621 & 0.3191 & 0.0241 & 0.0113 & 0.1016 & 0.0015 & 0.0066 & 0.0513 \\
\hline & & 3.1324 & 0.0011 & 0.0051 & 0.0676 & 0.0020 & 0.0191 & 0.0072 & 0.0435 \\
\hline & \multirow{2}{*}{ Phase B } & 3.1574 & 0.9173 & 0.0825 & 0.0171 & 0.3285 & 0.0018 & 0.0234 & 0.0752 \\
\hline & & 3.1303 & 0.0038 & 0.0074 & 0.0665 & 0.0045 & 0.0194 & 0.0106 & 0.0449 \\
\hline & \multirow{2}{*}{ Phase C } & 3.1582 & 0.9409 & 0.0852 & 0.0156 & 0.3382 & 0.0020 & 0.0238 & 0.0834 \\
\hline & & 3.1407 & 0.0039 & 0.0072 & 0.0285 & 0.0040 & 0.0189 & 0.0113 & 0.0426 \\
\hline
\end{tabular}

Table 1: Each MPCE of the third layer through GHM multiwavelet packet decomposition

According to the Table 1 the MPCEs of fault currents are significantly discriminated from the ones of normal currents that distributes more evenly, and the MPCEs have obvious difference when the faults are different. The results of simulation indicate that the MPCE can effectively withdraw the transient signals characteristics.

\section{Transient signals recognition algorithm based on RBF neural network}

\subsection{RBF neural network}

A typical BP neural network is a nonlinear regression technique that attempts to minimize the global error. Its training process includes forward and backward propagation, with the desired output used to generate error values for back propagation to iteratively improve the output. However, it is not suitable for certain applications such as the real-time control problems. BP is limited partly by the slow training performance, so
RBF neural network was developed instead.

The RBF neural network is different from BP with sigmoid activation functions utilizing basis functions in the hidden layer, which are locally responsive to input stimulus. These hidden nodes are usually implemented with a Gaussian kernel. Each hidden node in a RBF neural network has a radially symmetrical response around the center vector, and the output layer is a set of linear combiner with weights.

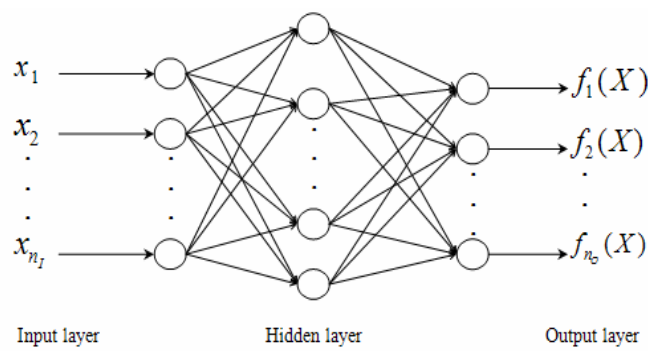

Fig. 4: Structure of RBF neural network.

The RBF network has a topology of one-hidden-layer. The input and output dimensions of the network are denoted by $n_{I}$ and $n_{O}$ respectively, and 
$n_{h}$ is the number of hidden nodes. The network structure is shown in Fig. 4. Each output node is a linear combiner defined by

$$
f_{i}(X)=\sum_{j=1}^{n_{h}} \Phi_{j}\left(\left\|X-C_{j}\right\|\right) \cdot \theta_{j i} \quad 1 \leq i \leq n_{O} \quad \text { (10) }
$$

Where

$$
\begin{aligned}
& X \in R^{n_{I}} \quad \text { input vector; } \\
& C_{j} \in R^{n_{I}} \quad \text { RBF centers; } \\
& \|.\| \quad \text { denotes the Euclidean norm; } \\
& \theta_{j i} \quad \text { weights. }
\end{aligned}
$$

\subsection{Principle of transient signals recognition algorithm}

After signals features are extracted effectively from transient signals according to multiwavelet packet coefficient entropy, the algorithm of transient signals recognition needs to be set up. First, a great deal of transient currents under various situations is sampled. Each phase current should be implemented $\mathrm{N}$ layers multiwavelet packet decomposition to. MPCEs of $2^{\mathrm{N}}$ frequency bands of the $N$ th layer of each phase current are calculated utilizing (7) (8). Then the RBF neural network is trained by constructing these MPCEs as the input eigenvector. The trained RBF neural network can recognize whether a 1-phase-to-ground, 2-phase-to-ground, phase-to-phase, 3-phase or lightning strokes fault has occurred. In addition, it can recognize the normal state.

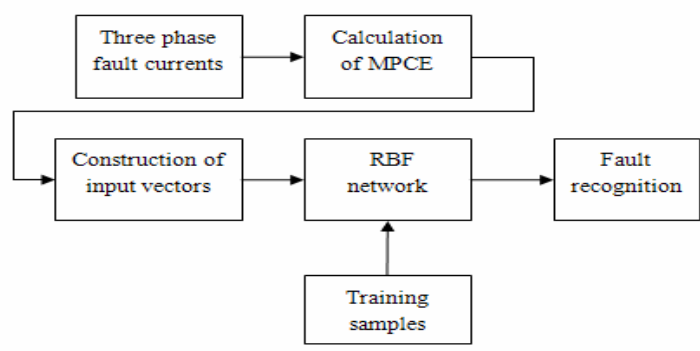

Fig. 5: Transient signals recognition process.

When a fault occurs on the transmission lines, the MPCEs of the sampled fault currents are extracted. Then by taking the MPCEs as the input vector of the trained RBF neural network the result of the fault type can be obtained.

\section{Digital simulation}

An extensive series of simulation studies has been carried out on the model system described earlier. To cover all regions of operating conditions, training data and testing data were generated from the system by varying: 1) The selected fault inception angles are increased in steps of $10^{\circ}$ from phase A voltage zero-crossing to $90^{\circ} ; 2$ ) The fault resistances are increased in steps of $50 \Omega$ from $0 \Omega$ to $300 \Omega ; 3$ ) Fault locations are varied in steps of $10 \%$ of line length from A-end to B-end. And on the simulation model different lightning currents are also set. Data window width is half power cycle of pre-fault and half power cycle of post-half.

Three methods based respectively on SA4 and GHM multiwavelet packet and DB4 traditional wavelet packet are adopted to implement transient signals recognition. In order to compare conveniently recognition capability of the three methods two indices are selected: 1) relative error of recognition results; 2) variance of recognition results.

Table 2 shows the recognition results of testing samples. Where: the inductive lightning 1 and 2 are inductive lightning strokes on the middle and end of the line respectively; non-fault lightning 1 and 2 are lightning strokes without faults on the middle and end of the line respectively; the lightning-induced severe fault is lightning strokes inducing severe grounding short-circuit (the fault inception angle is $90^{\circ}$ ) on the middle of the line; the lightning-induced weak fault is lightning strokes inducing weak grounding short-circuit (the fault inception angle is $5^{0}$ ). In the Table 2 ANN1 column represents recognition results of RBF neural network, which is trained by fault features extracted with SA4 multiwavelet packet and balance pre-processing [5]. The number of SA4 multiwavelet packet decomposition layers is 3 , that is to say $\mathrm{N}=3$. ANN2 column shows the ones with GHM multiwavelet packet and GHM.init pre-processing. The number of GHM multiwavelet packet decomposition layers is 3 . ANN3 column shows the ones with traditional Db4 wavelet packet, which filter length is equal to the one of SA4 multiwavelet packet. The number of DB4 wavelet packet decomposition layers is also 3 .

As shown in Table 2, recognition capability of RBF network trained by fault features extracted with SA4 and GHM multiwavelet packet is better than the one with DB4 wavelet packet. The main reason is that multiwavelet packet can provide more than one fold fault features than DB4 wavelet packet for RBF neural network, so the trained RBF neural network with SA4 and GHM multiwavelet packet is more sensitive to different transient signals than the one with DB4 wavelet packet. From Table 2 we can also find that the RBF neural network with multiwavelet packet is steadier than the one with traditional wavelet packet on recognizing different transient signals. 


\begin{tabular}{|c|c|c|c|c|c|c|c|c|c|}
\hline \multicolumn{4}{|c|}{ Testing samples } & \multicolumn{3}{|c|}{ Recognition results } & \multicolumn{3}{|c|}{ Relative error $/ \%$} \\
\hline Location/\% & Fault & Resistance $/ \Omega$ & Inception angle & ANN1 & ANN2 & ANN3 & ANN1 & ANN2 & ANN3 \\
\hline 18 & $\mathrm{AG}$ & 170 & $0^{\circ}$ & 1.0518 & 1.0136 & 0.9970 & 5.18 & 1.36 & -0.30 \\
\hline 18 & $\mathrm{ABC}$ & 14 & $0^{\circ}$ & 0.9988 & 0.9989 & 0.9938 & -0.12 & -0.11 & -0.62 \\
\hline 21 & AG & 120 & $12^{\circ}$ & 0.9819 & 0.9972 & 0.9990 & -1.81 & -0.28 & -0.10 \\
\hline 23 & $\mathrm{AB}$ & 22 & $12^{\circ}$ & 1.0028 & 0.9971 & 0.9871 & 0.28 & -0.29 & -1.29 \\
\hline 23 & $\mathrm{CG}$ & 240 & $19^{\circ}$ & 0.9702 & 1.0858 & 1.0790 & -2.98 & 8.58 & 7.90 \\
\hline 24 & $\mathrm{CA}$ & 75 & $19^{\circ}$ & 0.9966 & 1.0102 & 0.9784 & -0.34 & 1.02 & -2.16 \\
\hline 27 & BG & 290 & $31^{\circ}$ & 1.0283 & 0.9569 & 0.8683 & 2.83 & -4.31 & -13.17 \\
\hline 33 & $\mathrm{AB}$ & 80 & $31^{\circ}$ & 1.0192 & 1.0555 & 0.9592 & 1.92 & 5.55 & -4.08 \\
\hline 35 & $\mathrm{ABC}$ & 5 & $42^{\circ}$ & 1.0669 & 0.9326 & 1.0650 & 6.69 & -6.74 & 6.50 \\
\hline 48 & $\mathrm{CA}$ & 0 & $42^{\circ}$ & 1.0130 & 1.0408 & 0.9984 & 1.30 & 4.08 & -0.16 \\
\hline 51.5 & BG & 45 & $55^{\circ}$ & 1.0011 & 0.9635 & 0.9184 & 0.11 & -3.65 & -8.16 \\
\hline 55 & $\mathrm{AB}$ & 63 & $55^{\circ}$ & 0.9985 & 1.0225 & 1.0070 & -0.15 & 2.25 & 0.70 \\
\hline 62 & BCG & 80 & $63^{\circ}$ & 0.9866 & 0.9729 & 1.0563 & -1.34 & -2.71 & 5.63 \\
\hline 67 & AG & 7 & $63^{\circ}$ & 1.0220 & 0.9826 & 0.8772 & 2.20 & -1.74 & -12.28 \\
\hline \multirow{3}{*}{$\begin{array}{c}1.2 / 50 \mu \mathrm{s} \\
\text { Lightning } \\
\text { Current }\end{array}$} & \multicolumn{3}{|c|}{ Inductive lightning $1(\mathrm{I}=1 \mathrm{kA})$} & 0.9789 & 0.9863 & 0.9980 & -2.11 & -1.37 & -0.20 \\
\hline & \multicolumn{3}{|c|}{ Non-fault lightning $1(\mathrm{I}=3 \mathrm{kA})$} & 1.0222 & 0.9608 & 1.0724 & 2.22 & -3.92 & 7.24 \\
\hline & \multicolumn{3}{|c|}{ Lightning-induced severe fault (I=50 kA) } & 0.9192 & 0.9269 & 1.1001 & -8.08 & -7.31 & 10.01 \\
\hline \multirow{3}{*}{$\begin{array}{c}5 / 100 \mu \mathrm{s} \\
\text { Lightning } \\
\text { Current }\end{array}$} & \multicolumn{3}{|c|}{ Inductive lightning $2(\mathrm{I}=3 \mathrm{kA})$} & 0.9861 & 0.9522 & 0.9933 & -1.39 & -4.78 & -0.67 \\
\hline & \multicolumn{3}{|c|}{ Non-fault lightning $2(\mathrm{I}=5 \mathrm{kA})$} & 1.0128 & 0.9901 & 1.0041 & 1.28 & -0.99 & 0.41 \\
\hline & \multicolumn{3}{|c|}{ Lightning-induced weak fault (I=20 kA) } & 0.9974 & 1.0849 & 1.0144 & -0.26 & 8.49 & 1.44 \\
\hline \multicolumn{4}{|c|}{ Variance } & 0.0181 & 0.0380 & 0.0705 & - & - & - \\
\hline
\end{tabular}

Table 2: Recognition results with three methods and comparison.

Fig. 6 shows the comparison curves of relative error of recognition results with three methods. We can find that the relative error of SA4 multiwavelet packet is almost less than $\pm 5 \%$, and the relative error of DB4 wavelet packet is the greatest in three methods. The recognition results of RBF network with multiwavelet packet are more precise than the one with traditional wavelet packet.

\section{Conclusions}

The paper presents a novel method of transient signals recognition based on multiwavelet packet coefficient entropy and RBF neural network. The simulation results show that the RBF neural network trained by multiwavelet packet is able to recognize different transient signals correctly under various conditions and the recognition capability of RBF neural network trained by multiwavelet packet is better than the one trained by traditional wavelet.

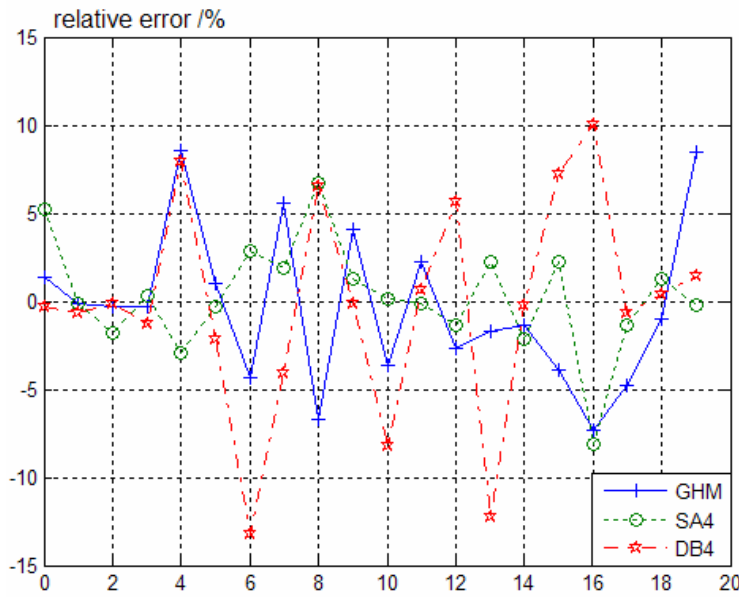

Fig. 6: The comparison of relative error of recognition results with three methods.

\section{Acknowledgement}

This work is supported by Fok Ying Tung Education 
Foundation (No. 101060) and Sichuan Province Distinguished Scholars Fund (No. 07ZQ026-012).

\section{References}

[1] X.N. Lin, P. Liu, C.M. Yang, A Superimposed Phase Selector Based on Symmetrical Components and Correlation Analysis. Proceedings of the CSEE, 22(5): 16-25.

[2] J.A. Jiang, C.S. Chen, C.W. Liu, a new protection scheme for fault detection, direction discrimination, classification, and location in transmission lines. IEEE Trans. Power Delivery, 18(1): 34-42, 2003.

[3] Z.G. Liu, Y.D. Zeng, Q.Q. Qian, De-noising of Electric Power System Signals Based on Different Multiwavelets. Proceedings of the CSEE, vol. 24, no. 1, pp. 30-34, 2004.

[4] Z.G. Liu, Q.Q. Qian, Compression of Fault Transient Data in Electric Power System Based on Multiwavelet. Proceedings of the CSEE, 23(10): 22-26, 2003.

[5] Z.G. Liu, Z.Y. He, Q.Q. Qian, A Fault Signal Data Compression Plan Based on Optimal Pre-processing Method of Multiwavelet. Power System Technology, 29(11): 40-43, 2005.

[6] B.Y. Xu,C.J. Huang, Y. Qian, X.C. Jiang, Application of Multiwavelet Based on Neighboring Coefficient Method In De-noising of Partial Discharge. Power System Technology, 29(15): 61-65, 2005.

[7] Z.G. Liu, D.B. Zhang, D.D. Ma, De-noising and Compression of Power Fault Signals Based on Best Multiwavelet Packet. 2005 IEEE/PES Transmission and Distribution Conference \& Exhibition: Asia and Pacific Dalian, China.
[8] M.B. Martin, Amy E.Bell, New Image Compression Techniques Using Multiwavelets and Multiwavelet Packets. IEEE Trans. Image Processing, 10(4): 500-510, 2001.

[9] W.J. Li, Y.C. LI, Fault Detection of Gearing System Based on Multiwavelet Packets. Proceedings of the Third International Conference on Machine Learning and Cybernetics, Shanghai, pp. 26-29, 2004.

[10]D. V. Coury and D. C. Jorge, Artificial neural network approach to distance protection of transmission lines. IEEE Trans. Power Delivery, 13(1): 102-108, 1998.

[11] T. S. Sidhu, H. Singh, and M. S. Sachdev, Design implementation and testing of an artificial neural network based fault direction discriminator for protecting transmission lines. IEEE Trans. Power Delivery, 10(2): 697-706, 1995.

[12] W.M. Lin, C.D. Yang and J.H. Lin, A Fault Classification method by RBF Neural Network with OLS Learning Procedure. IEEE Trans. Power Delivery, 16(4): 473-477, 2001.

[13]B. Zhang, Z.Y. He, Q.Q. Qian, application of wavelet entropy and adaptive nerve-fuzzy inference to fault classification. IEEE International Conference Power System Technology, 2006.

[14]Z.Y. He, Y.M. Cai, Q.Q. Qian, A Study of Wavelet Entropy Theory and Its Application in Electric Power System Fault Detection. Proceedings of the CSEE, 25(5): 38-43, 2005.

[15] Y.F. Ren, J.D. Duan, B.H. Zhang, Identification of Lightning Disturbance in Ultra-High-Speed Transmission Line Protection. 2005 IEEE/PES Transmission and Distribution Conference \& Exhibition: Asia and Pacific Dalian, China,2005. 